\title{
The effect of light vs dark coat color on thermal status in
}

\section{Labrador Retriever dogs.}

Authors:

9 Caitlin Neander ${ }^{1}$, Janice Baker ${ }^{2}$, Kathleen Kelsey ${ }^{3}$, Jean Feugang ${ }^{4}$, Erin Perry ${ }^{1 *}$

11 Affiliations:

$12{ }^{1}$ Department of Animal Science, Food and Nutrition, College of Agriculture Science, Southern

13 Illinois University, Carbondale, IL

$14 \quad{ }^{2}$ Veterinary Tactical Group, Vass, NC 28394

15

16

17

${ }^{3}$ Working Dog Enterprises, Sturgeon, MO 65284

${ }^{4}$ Department of Animal and Dairy Science, College of Agriculture and Life Sciences, Mississippi

19 State University, Mississippi State, MS

* Corresponding author: erin.perry@siu.edu 


\section{Abstract}

Although dark coat color in dogs has been theorized as a risk factor for thermal stress,

31 there is little evidence in the scientific literature to support that position. We utilized 16 nonconditioned Labradors ( 8 black and 8 yellow) in a three-phase test to examine effects of coat color on thermal status of the dog. Rectal, gastrointestinal (GI), surface temperature, and respiration rate measured in breaths per minute $(\mathrm{bpm})$, were collected prior to (Baseline — phase 1) and immediately after a controlled 30-minute walk in an open-air environment on a sunny day (Sunlight — phase 2). Follow up measurements were taken 15 minutes after walking (Cool

37 down - phase 3) to determine post-exposure return to baseline. No effect of coat color was measured for rectal, gastrointestinal or surface temperature, or respiration $(\mathrm{P}>0.05)$ in dogs following their 30-minute walk. Temperatures increased similarly across both coat colors (rectal measured $(\mathrm{P}>0.05)$. These data contradict the commonly held theory that dogs with darker coat color may experience a greater thermal change when exposed to direct sunlight compared to

47 dogs with a lighter coat color.

Keywords: Canine, Labradors, Thermal Stress, Heat Stress, Dog, Thermal Imaging,

\section{Temperature, Coat Color}




\section{Introduction}

Darker coat color has been suggested as a potential risk factor for heat injury in dogs in several publications [1-4] However, little evidence is available to support this theory, and a majority of these claims appear in the introduction or discussion sections of publications, or in review articles, with no supporting data. One study in Greyhounds reported higher rectal the darker coat participant group. These males were significantly larger in size than their female cohorts, so it is not known if sex or size played a role in their results. In addition, the darker colored group $(n=166)$ had more than twice the number of the light coated group $(n=63)$ which may have impacted the outcome [5]. In another study using Newfoundland dogs, researchers tested patches of white and black fur exposed to heat lamps. Authors measured the microclimate of the dog's coat and reported no significant difference in temperature between white and black fur regions on the dogs [6]. However, this study did not examine two separate groups of dogs with single coat colors (i.e. solid black or white).

Work in cattle has demonstrated an impact on thermal status associated with coat color, but this has not been thoroughly investigated in dogs. Increased solar absorption in darker coated cattle has been demonstrated to increase overall heat gain [7]. Darker cattle exposed to direct sunlight had a surface temperature gain of $4.8^{\circ} \mathrm{C}$, while lighter cattle only increased surface temperature by $0.7^{\circ} \mathrm{C}$ [8]. Additionally, this study reported increased incidence of elevated surface temperature, respiration, sweating, and heat stress signals in darker colored cattle compared to lighter colored cattle.

The risk of thermal injury to dogs is of significant concern to the veterinary community and is considered a common occurrence especially during the summer months. Evidence to validate the 
74 ideas surrounding coat color as a risk factor would be helpful in establishing a better understanding of

75 any increased danger facing dark coated dogs. Assessment of risk for heat injury can only be

76 accurately evaluated by studying dogs that incur heat injury in comparison to dogs that do not,

77 whether prospectively or retrospectively. Prospective studies of this nature are inherently difficult to conduct as our current standards of ethics and animal stewardship generally preclude experimentally induced heat injury in dogs. In addition, given the relatively low incidence of naturally occurring heat injury in any given population of dogs, prospective studies relying on naturally occurring cases would

81 require a significant amount of time to complete. Thus, risk of heat injury is primarily based on

82 observations of normal thermoregulatory reactions to safe levels of thermal stress, typically induced

83 by exercise. In this study, we exposed dogs of light and dark coat colors to mild exercise (i.e. loose

84 leash walk) in direct sunlight to assess thermoregulatory reactions and measure various parameters associated with body temperature and thermoregulation. The objective of this research was to identify the impact of coat color on the thermal status of dogs exposed to direct sunlight and to measure the increase in temperature experienced by black dogs as compared to yellow dogs.

90 Animals and Diets

Southern Illinois University prior to initiation of the study. The study was conducted in mid- 
group turnout but the absence of a specific conditioning or exercise program. All dogs utilized came from 2 litters to limit for genetic variability. Dogs had a mean age of $2.73 \pm 1.86$ years, mean weight $26.6 \pm 3.32 \mathrm{~kg}$ and a mean BCS of $5.5 \pm 1.5$. Study participants were maintained on a commercial kibble diet (Victor High Energy, Mid America Pet Food Mount Pleasant, Texas) and fed twice daily for 60 days prior to the study. All dogs were up to date on vaccinations (rabies, bordetella, DHLPP) and received a monthly standardized parasite control regimen (Frontline Plus, Merial France) (Interceptor Plus, Elanco, Greenfield, IN). All study participants received a health screening by a licensed veterinarian prior to inclusion in the study and were also assigned a body condition score (BCS) by a trained researcher (Nestle Purina Petcare Company, St. Louis, MO). Following this exam, one canine was excluded from participation due to a previously undiagnosed dermal condition. Dogs of opposite colors were paired according to sex and BCS for participation.

\section{Phases}

The study was separated into three phases. Phase 1 (Baseline) included housing of each dog for 30 uninterrupted minutes in a climate-controlled room in individual crates. Phase 2 (Sunlight) consisted of 30 minutes of loose leash walking at a controlled pace in an uncovered outdoor sandy arena measuring $30 \mathrm{~m}$ by $60 \mathrm{~m}$. The study concluded with Phase 3 (Cooling) and incorporated a 15minute rest in a climate-controlled room in individual crates. All dogs were monitored throughout the study by veterinary staff stationed in the center of the outdoor arena and climate-controlled holding area, and all dogs were allowed ad libitum access to water while in their crates during both the Baseline and Cooling phases of the study.

Environmental conditions in the outdoor arena and in the climate-controlled room were monitored (Accurite Wireless Weather Station, Chaney Instrument Co. Lake Geneva, WI) to record temperature, humidity and heat index every five minutes. 


\section{Data Collection}

Thermal status data for each dog were captured immediately following each of the three

122 phases utilizing four methods as shown in Fig 1. Gastrointestinal (GI) data were captured using an

123 ingestible thermistor orally administered (CorTemp, CorTemp Inc, Palmetto, FL) 30 minutes

$124( \pm 15)$ prior to the Baseline phase. GI temperatures were monitored with a handheld wireless reader (CorTemp, HQInc Palmetto, FL.). GI temperature was recorded in triplicate for each data

126 collection period to ensure accuracy and the mean was utilized for statistical analysis. Rectal

127 measurements were collected in tandem, using calibrated, 8second digital thermometers

128 (American Diagnostics Company ADTEMP II model \#413B) inserted to a depth of

129 approximately $2 \mathrm{~cm}$ with petroleum jelly to minimize canine discomfort. Thermal images of

130 participants were captured using a forward-looking infrared thermal camera (FLIR T400 thermal

131 camera) at an approximate distance of 2 meters from the canine to capture body surface

132 temperature as previously described [9-12]. To reduce the effects of environmental factors, all

133 images were captured in an enclosed area with no exposure to wind or direct sunlight. Thermal

134 images were analyzed using thermography software (ThermaCam Researcher Professional 2.9,

135 FLIR Systems Inc. Wilson, OR, USA) to determine body surface temperature at the left eye

136 and caudal abdomen as described previously [13-15] with examples shown in Fig 2.

137

138

139

140

Fig 1. Pictogram representing the phases and points of data collection.

Fig 2. Thermography ${ }^{1}$ depicting Baseline ${ }^{2}$ (left) and Sunlight ${ }^{3}$ (right) values for Labrador Black 6 . The two areas of interest were left eye ${ }^{4}$ and left caudal abdomen ${ }^{5}$. 
147 monitoring. The head, face, and tongue were captured and later played back in slow motion to

148 count respiration during this 30 second period. A single independent observer was utilized

149 throughout all canine respiration videos to minimize observer bias. Respiration was calculated as

150 breaths per minute $(\mathrm{BPM})=30$-second respiration $\mathrm{x} 2$.

\section{Statistical Analysis}

All data were analyzed using SAS version 9.4 (SAS Institute Inc., Cary, NC). Each

153 phase (baseline, sunlight, cooling) was examined using a Proc Glm repeated measures test.

154 Baseline and Sunlight temperatures were examined using a paired t test to identify main effects

155 of coat color and sex for dependent variables including rectal, gastrointestinal, surface

156 temperature, respiration rate, and water consumption.

157 Additionally, a multivariate ANOVA was utilized to identify differences associated with the

158 interactions of coat color and sex on rectal, gastrointestinal, body surface temperature,

159 respiration, and water consumption. Water consumption throughout the data collection period

160 was calculated as: Water offered - Water remaining $=$ Water consumed

161 Return to baseline was identified as having achieved a cooling phase temperature within $0.5^{\circ} \mathrm{F}$ of the

162 dog's initial baseline temperature using the below equations. If the cooling phase temperature had

163 fallen to within $0.5^{\circ} \mathrm{F}$, it was deemed "yes" the dog returned to a baseline temperature. The following

164 equation was utilized:

167 Return to baseline was reported as Yes or No and was analyzed using the Proc Freq procedure of SAS

168 (chi square) to examine differences coat color and sex. Significance for all outcomes was established

169 at $\mathrm{P}<0.05$. 


\section{$171 \quad$ Results}

173 black dogs, and $1.83{ }^{\circ} \mathrm{C}$ in yellow coated dogs $(\mathrm{P}<0.0001)$. Similarly, GI temperatures

174 increased by $1.89{ }^{\circ} \mathrm{C}$ in black coated dogs, and $1.94{ }^{\circ} \mathrm{C}$ in the yellow group, $(\mathrm{P}<0.0001)$ as

175 shown in Fig 3. Eye surface temperature increased by $2.8^{\circ} \mathrm{C}$ black and $1.93{ }^{\circ} \mathrm{C}$ yellow $(\mathrm{P}<$

176 0.005) and abdominal surface temperature increased by $2.93{ }^{\circ} \mathrm{C}$ black and $2.35{ }^{\circ} \mathrm{C}$ yellow $(\mathrm{P}<$

177 0.0001). See Figs 3-4, Table 1. No significant temperature difference was noted between black

178 and yellow Labradors across all phases.

179 Fig 3. Mean change in rectal $^{1}$ and gastrointestinal temperature (GI) ${ }^{2}$ across three phases in non-

180 conditioned Labradors.

182 Fig 4. Mean change in body surface temperature measured by thermography ${ }^{1}$ at the eye ${ }^{2}$ and 183 abdomen ${ }^{3}$ in non-conditioned Labradors

185 Table 1. Mean values of thermal status indicators ${ }_{1}$ across three phases (Baseline ${ }^{2}$, Sunlight $^{3}$, Cooling $^{4}$ ) in 186 Labradors grouped by coat color.

\begin{tabular}{|c|c|c|c|c|c|c|c|}
\hline Variable & Color & Baseline & P-value & Sunlight & P-value & Cooling & P-value \\
\hline \multirow[t]{2}{*}{ Rectal $_{5}$} & Black & $\begin{array}{l}38.44 \pm 0.37^{\circ} \mathrm{C} \\
101.2 \pm 0.7^{\circ} \mathrm{F}\end{array}$ & \multirow[t]{2}{*}{0.8404} & $\begin{array}{l}40.3 \pm 0.41^{\circ} \mathrm{C}^{\mathrm{b}} \\
104.5 \pm 0.8^{\circ} \mathrm{F}\end{array}$ & \multirow[t]{2}{*}{0.9354} & $\begin{array}{l}39.46 \pm 0.34 \\
{ }^{\circ} \mathrm{C}^{\mathrm{c}} \\
103 \pm 0.6^{\circ} \mathrm{F}\end{array}$ & \multirow[t]{2}{*}{0.4673} \\
\hline & Yellow & $\begin{array}{l}38.51 \pm 0.52^{\circ} \mathrm{C} \\
\text { a } \\
101.3 \pm 1.0^{\circ} \mathrm{F}\end{array}$ & & $\begin{array}{l}40.31 \pm 0.34^{\circ} \mathrm{C} \\
\mathrm{b} \\
104.6 \pm 0.6^{\circ} \mathrm{F}\end{array}$ & & $\begin{array}{l}39.43 \pm 0.30 \\
{ }^{\circ} \mathrm{C}^{\mathrm{c}} \\
102.8 \pm 0.4{ }^{\circ} \mathrm{F}\end{array}$ & \\
\hline \multirow[t]{2}{*}{$\mathbf{G I}_{6}$} & Black & $\begin{array}{l}38.76 \pm 0.25^{\circ} \mathrm{C} \\
101.8 \pm 0.5^{\circ} \mathrm{F}\end{array}$ & \multirow[t]{2}{*}{0.6279} & $\begin{array}{l}40.68 \pm 0.47^{\circ} \mathrm{C} \\
\mathrm{b} \\
105.2 \pm 0.9^{\circ} \mathrm{F}\end{array}$ & \multirow[t]{2}{*}{0.8286} & $\begin{array}{l}39.2 \pm 0.6^{\circ} \mathrm{C}^{\mathrm{c}} \\
102.6 \pm 1.1^{\circ} \mathrm{F}\end{array}$ & \multirow[t]{2}{*}{0.8153} \\
\hline & Yellow & $\begin{array}{l}38.66 \pm 0.48^{\circ} \mathrm{C} \\
\mathrm{a} \\
101.6 \pm 0.9^{\circ} \mathrm{F}\end{array}$ & & $\begin{array}{l}40.61 \pm 0.21^{\circ} \mathrm{C} \\
\mathrm{b} \\
105.1 \pm 0.4^{\circ} \mathrm{F}\end{array}$ & & $\begin{array}{l}39.29 \pm 0.26 \\
{ }^{\circ} \mathrm{C}^{\mathrm{c}} \\
102.6 \pm 0.5 \\
{ }^{\circ} \mathrm{C}^{\mathrm{c}}\end{array}$ & \\
\hline $\mathbf{E y e}_{7}$ & Black & $\begin{array}{l}36.09 \pm 0.57^{\circ} \mathrm{C} \\
\text { a } \\
97.0 \pm 1.1^{\circ} \mathrm{F}\end{array}$ & 0.0934 & $\begin{array}{l}38.89 \pm 0.42{ }^{\circ} \mathrm{C} \\
\text { b } \\
102.0 \pm 0.8^{\circ} \mathrm{F}\end{array}$ & 0.3004 & $\begin{array}{l}37.4 \pm 0.5^{\circ} \mathrm{C}^{\mathrm{c}} \\
99.3 \pm 0.96^{\circ} \mathrm{F}\end{array}$ & 0.0973 \\
\hline
\end{tabular}




\begin{tabular}{|c|c|c|c|c|c|c|c|}
\hline & Yellow & $\begin{array}{l}36.68 \pm 0.55^{\circ} \mathrm{C} \\
\text { a } \\
98.0 \pm 1.1^{\circ} \mathrm{F}\end{array}$ & & $\begin{array}{l}38.6 \pm 0.54^{\circ} \mathrm{C}^{b} \\
101.5 \pm 1.0^{\circ} \mathrm{F}\end{array}$ & & $\begin{array}{l}36.9 \pm 0.51 \\
{ }^{\circ} \mathrm{C}^{\mathrm{c}} \\
98.4 \pm 1.0^{\circ} \mathrm{F}\end{array}$ & \\
\hline \multirow[t]{2}{*}{ Abdominal $_{8}$} & Black & $\begin{array}{l}36.24 \pm 0.91^{\circ} \mathrm{C} \\
\text { a } \\
97.2 \pm 1.7^{\circ} \mathrm{F}\end{array}$ & \multirow[t]{2}{*}{0.7763} & $\begin{array}{l}39.15 \pm 0.73^{\circ} \mathrm{C} \\
\text { b } \\
102.5 \pm 1.4^{\circ} \mathrm{F}\end{array}$ & \multirow[t]{2}{*}{0.0908} & $\begin{array}{l}37.4 \pm 0.5^{\circ} \mathrm{C}^{\mathrm{c}} \\
100.7 \pm 1.3^{\circ} \mathrm{F}\end{array}$ & \multirow[t]{2}{*}{0.1002} \\
\hline & Yellow & $\begin{array}{l}36.01 \pm 1.44^{\circ} \mathrm{C} \\
\text { a } \\
96.9 \pm 2.9^{\circ} \mathrm{F}\end{array}$ & & $\begin{array}{l}38.4 \pm 0.75^{\circ} \mathrm{C}^{\mathrm{b}} \\
101.1 \pm 1.5^{\circ} \mathrm{F}\end{array}$ & & $\begin{array}{l}36.9 \pm 0.51 \\
{ }^{\circ} \mathrm{C}^{\mathrm{c}} \\
99.6 \pm 1.2{ }^{\circ} \mathrm{F}\end{array}$ & \\
\hline \multirow[t]{2}{*}{ Respiration $_{9}$} & Black & $123.3 \pm 20.5^{\mathrm{a}}$ & \multirow[t]{2}{*}{0.7206} & $270.5 \pm 40.2^{\mathrm{b}}$ & \multirow[t]{2}{*}{0.744} & $241.7 \pm 24.5^{\mathrm{c}}$ & \multirow[t]{2}{*}{0.2998} \\
\hline & Yellow & $132.9 \pm 40.7^{\mathrm{a}}$ & & $276.6 \pm 28.2^{\mathrm{b}}$ & & $216.4 \pm 59.7^{\mathrm{c}}$ & \\
\hline
\end{tabular}

187

188

189

190

191

192

193

194

195

196

197

198

199

200

201

202

203

204

205

206

207

208

209

210

211

212

213

${ }^{*}$ Notes a significant difference observed by coat color

a,b,c Notes a significant difference observed by phase

${ }^{1}$ Thermal status indicators including rectal, gastrointestinal, eye and abdominal temperature

${ }^{2}$ Baseline occurred 30 minutes prior to sunlight exposure (sunlight) and recorded initial measurements

${ }^{3}$ Sunlight phase consisted of 30 minutes of active walking in a sunny outdoor area on a leash

${ }^{4}$ Cooling phase occurred 15 minutes after walking in a climate-controlled room with water

${ }^{5}$ Rectal temperature was recorded by inserting thermometer to a depth of approximately $2 \mathrm{~cm}$ with petroleum jelly

${ }^{6} \mathrm{GI}$ (gastrointestinal) temperature was recorded with an ingestible thermistor CorTemp 30 minutes prior to baseline

${ }^{7}$ Eye temperature was captured using thermography FLIR T400 at the left eye

${ }^{8}$ Abdominal temperature was captured using thermography FLIR T400 at the left caudal abdomen

${ }^{9}$ Respiration rate was captured for 30 seconds utilizing a GoPro, depicted as breaths per minute (bpm)

Similarly, all temperature measurements significantly decreased from sunlight to cooling

phase. Following cessation of cooling phase, rectal temperatures decreased by $0.84^{\circ} \mathrm{C}$ black and 1.0

${ }^{\circ} \mathrm{C}$ yellow $(\mathrm{P}<0.0001)$ and GI temperatures decreased $1.45 \mathrm{C}$ in black dogs and 1.33 in yellow

dogs $(\mathrm{P}<0.0001)$ as shown in Fig 3. Thermal eye surface temperature decreased $1.49^{\circ} \mathrm{C}$ in black

and $1.71{ }^{\circ} \mathrm{C}$ in yellow dogs $(\mathrm{P}<0.005)$, and abdominal surface temperature decreased by $1.0{ }^{\circ} \mathrm{C}$

black and $0.85^{\circ} \mathrm{C}$ yellow $(\mathrm{P}<0.0001)$ as shown in Fig 4 . A similar change in respiration rates

was shown across both coat colors of Labradors, meaning that coat color did not significantly

impact breathing rates across phases $(\mathrm{P}>0.05)$, as shown in Fig 5 .

Fig 5. Mean change in respiration rates ${ }^{1}$ across three phases in non-conditioned Labradors. 
215 surface $\left(2.41^{\circ} \mathrm{C}\right)$ and abdominal surface $\left(2.67^{\circ} \mathrm{C}\right)$ temperatures of all dogs when Baseline and

216 Sunlight temperatures were compared $(\mathrm{P}<0.0001)$. Furthermore, returning to the climate-

217 controlled room significantly decreased the rectal $\left(0.7^{\circ} \mathrm{C}\right)$, GI $\left(1.41^{\circ} \mathrm{C}\right)$, eye surface $\left(1.58^{\circ} \mathrm{C}\right)$, and

218 abdominal surface $\left(0.92^{\circ} \mathrm{C}\right)$ temperature of all dogs $(\mathrm{P}<0.0001)$.

219

220

221

222

223

224

225

226

227

228

229

230

231

232

233

234

235

After completion of 30 minutes in direct sunlight walking, both males and females saw a similar

increase across all temperatures, rectal $\left(1.83^{\circ} \mathrm{C}\right.$ male, $1.84^{\circ} \mathrm{C}$ female $), \mathrm{GI}\left(2.04^{\circ} \mathrm{C}\right.$ male, $1.87^{\circ} \mathrm{C}$

female), eye surface $\left(2.3^{\circ} \mathrm{C} 2\right.$ male, $2.5^{\circ} \mathrm{C}$ female $)$ and abdominal surface $\left(2.93^{\circ} \mathrm{C}\right.$ male and 2.5

${ }^{\circ} \mathrm{C}$ female). A similar fall in temperatures for both sexes was seen after 15 minutes of passive

cooling, rectal $\left(0.92^{\circ} \mathrm{C}\right.$ male, $0.89^{\circ} \mathrm{C}$ female $)$, GI $\left(1.32^{\circ} \mathrm{C}\right.$ male, $1.47^{\circ} \mathrm{C}$ female $)$, eye surface

(1.62 ${ }^{\circ} \mathrm{C}$ male, $1.57^{\circ} \mathrm{C}$ female), and abdominal surface $\left(1.0^{\circ} \mathrm{C}\right.$ male, $0.87^{\circ} \mathrm{C}$ female $)$ as shown in

Figures 6-7, Table 2.

Fig 6. Mean change in $\operatorname{rectal}^{1}$ and gastrointestinal temperature $(\mathrm{GI})^{2}$ across three phases in nonconditioned Labradors.

Fig 7. Mean change in surface temperature measured by thermography ${ }^{1}$ at the eye $e^{2}$ and abdomen $^{3}$ in non-conditioned Labradors across three phases.

\begin{tabular}{|c|c|c|c|c|c|c|c|}
\hline VARIABLE & SEX & BASELINE & $\begin{array}{l}\text { P- } \\
\text { VALUE }\end{array}$ & SUNLIGHT & P-VALUE & COOLING & $\begin{array}{l}\text { P- } \\
\text { VALUE }\end{array}$ \\
\hline \multirow[t]{2}{*}{ RECTAL $_{5}$} & Male & $\begin{array}{l}38.52 \pm 0.35^{\circ} \mathrm{C}^{\mathrm{a}} \\
101.3 \pm 0.7^{\circ} \mathrm{F}\end{array}$ & \multirow[t]{2}{*}{0.8536} & $\begin{array}{l}40.35 \pm 0.31^{\circ} \mathrm{C}^{b} \\
104.6 \pm 0.6^{\circ} \mathrm{F}\end{array}$ & \multirow[t]{2}{*}{0.6991} & $\begin{array}{l}39.43 \pm 0.3^{\circ} \mathrm{C}^{\mathrm{c}} \\
103 \pm 0.6^{\circ} \mathrm{F}\end{array}$ & \multirow[t]{2}{*}{0.7511} \\
\hline & Female & $\begin{array}{l}38.44 \pm .5^{\circ} \mathrm{C}^{\mathrm{a}} \\
101.2 \pm 0.9^{\circ} \mathrm{F}\end{array}$ & & $\begin{array}{l}40.28 \pm 0.41^{\circ} \mathrm{C}^{b} \\
104.5 \pm 0.8^{\circ} \mathrm{F}\end{array}$ & & $\begin{array}{l}39.39 \pm 0.27^{\circ} \mathrm{C}^{\mathrm{c}} \\
102.9 \pm 0.5^{\circ} \mathrm{F}\end{array}$ & \\
\hline \multirow[t]{2}{*}{$\mathbf{G I}_{6}$} & Male & $\begin{array}{l}38.68 \pm 0.36^{\circ} \mathrm{C}^{\mathrm{a}} \\
101.7 \pm 0.7^{\circ} \mathrm{F}\end{array}$ & \multirow[t]{2}{*}{0.9407} & $\begin{array}{l}40.72 \pm 0.21^{\circ} \mathrm{C}^{b} \\
105.3 \pm 0.4^{\circ} \mathrm{F}\end{array}$ & \multirow[t]{2}{*}{0.6005} & $\begin{array}{l}39.4 \pm 0.41^{\circ} \mathrm{C}^{\mathrm{c}} \\
102.9 \pm 0.8^{\circ} \mathrm{F}\end{array}$ & \multirow[t]{2}{*}{0.3736} \\
\hline & Female & $\begin{array}{l}38.73 \pm 0.39^{\circ} \mathrm{C}^{\mathrm{a}} \\
101.7 \pm 0.7^{\circ} \mathrm{F}\end{array}$ & & $\begin{array}{l}40.6 \pm 0.44^{\circ} \mathrm{C}^{\mathrm{b}} \\
105.1 \pm 0.9^{\circ} \mathrm{F}\end{array}$ & & $\begin{array}{l}39.13 \pm 0.49^{\circ} \mathrm{C}^{\mathrm{c}} \\
102.4 \pm 0.9^{\circ} \mathrm{F}\end{array}$ & \\
\hline
\end{tabular}




\begin{tabular}{|c|c|c|c|c|c|c|c|}
\hline \multirow[t]{2}{*}{$\mathrm{EYE}_{7}$} & Male & $\begin{array}{l}36.58 \pm 0.64^{\circ} \mathrm{C}^{\mathrm{a}} \\
97.9 \pm 1.3^{\circ} \mathrm{F}\end{array}$ & \multirow[t]{2}{*}{0.2509} & $\begin{array}{l}38.9 \pm 0.14^{\circ} \mathrm{C}^{\mathrm{b}} \\
102.0 \pm 0.3^{\circ} \mathrm{F}\end{array}$ & \multirow[t]{2}{*}{0.3087} & $\begin{array}{l}37.28 \pm 0.5^{\circ} \mathrm{C}^{\mathrm{c}} \\
99.1 \pm 1.0^{\circ} \mathrm{F}\end{array}$ & \multirow[t]{2}{*}{0.5457} \\
\hline & Female & $\begin{array}{l}36.16 \pm 0.57^{\circ} \mathrm{C}^{\mathrm{a}} \\
97.1 \pm 1.1^{\circ} \mathrm{F}\end{array}$ & & $\begin{array}{l}38.66 \pm 0.62^{\circ} \mathrm{C}^{b} \\
101.6 \pm 1.2^{\circ} \mathrm{F}\end{array}$ & & $\begin{array}{l}37.09 \pm 0.59^{\circ} \mathrm{C}^{\mathrm{c}} \\
98.8 \pm 1.1^{\circ} \mathrm{F}\end{array}$ & \\
\hline \multirow[t]{2}{*}{ ABDOMINAL $_{8}$} & Male & $\begin{array}{l}36.2 \pm 0.95^{\circ} \mathrm{C}^{\mathrm{a}} \\
97.2 \pm 2.0^{\circ} \mathrm{F}\end{array}$ & \multirow[t]{2}{*}{0.8360} & $\begin{array}{l}39.13 \pm 0.34{ }^{\circ} \mathrm{C}^{\mathrm{b}} \\
102.4 \pm 0.7^{\circ} \mathrm{F}\end{array}$ & \multirow[t]{2}{*}{0.1674} & $\begin{array}{l}38.13 \pm 0.45^{\circ} \mathrm{C}^{\mathrm{c}} \\
100.6 \pm 0.9^{\circ} \mathrm{F}\end{array}$ & \multirow[t]{2}{*}{0.9533} \\
\hline & Female & $\begin{array}{l}36.09 \pm 1.33^{\circ} \mathrm{C}^{\mathrm{a}} \\
97.0 \pm 2.5^{\circ} \mathrm{F}\end{array}$ & & $\begin{array}{l}38.58 \pm 0.97^{\circ} \mathrm{C}^{b} \\
101.4 \pm 1.9^{\circ} \mathrm{F}\end{array}$ & & $\begin{array}{l}37.71 \pm 0.81^{\circ} \mathrm{C}^{\mathrm{c}} \\
99.9 \pm 1.5^{\circ} \mathrm{F}\end{array}$ & \\
\hline \multirow[t]{2}{*}{ RESPIRATION $_{9}$} & Male & $124.0 \pm 50.3^{\mathrm{a}}$ & \multirow[t]{2}{*}{0.8203} & $274.0 \pm 34.4^{b}$ & \multirow[t]{2}{*}{0.9533} & $225.3 \pm 33.9^{c}$ & \multirow[t]{2}{*}{0.8553} \\
\hline & Female & $130.2 \pm 51.4^{\mathrm{a}}$ & & $272.9 \pm 35.9^{b}$ & & $230.1 \pm 56.0^{c}$ & \\
\hline
\end{tabular}

236

237

238

239

240

241

242

243

244

245

246

247

248

249

250

251

252

253

254

255

256

257

258

259

260

261

262

263

264

${ }^{*}$ Notes a significant difference observed by sex

a,b,c Notes a significant difference observed by phase

${ }^{1}$ Thermal status indicators including rectal, gastrointestinal, eye and abdominal temperature

${ }^{2}$ Baseline occurred 30 minutes prior to sunlight exposure (sunlight) and recorded initial measurements

${ }^{3}$ Sunlight phase consisted of 30 minutes of active walking in a sunny outdoor area on a leash

${ }^{4}$ Cooling phase occurred 15 minutes after walking in a climate-controlled room with water

${ }^{5}$ Rectal temperature was recorded by inserting a thermometer rectally $2 \mathrm{~cm}$

${ }^{6} \mathrm{GI}$ (gastrointestinal) temperature was recorded with an ingestible thermistor CorTemp 30 minutes prior to baseline

${ }^{7}$ Eye temperature was captured using thermography FLIRT400 at the left eye

${ }^{8}$ Abdominal temperature was captured using thermography FLIR T400 at the left caudal abdomen

${ }^{9}$ Respiration rate was captured for 30 seconds utilizing a GoPro, depicted as breaths per minute(bpm)

Across all phases of the study, sex did not show a significant effect on respiration rates

of the Labradors, with both sexes showing a similar increase and decrease in bpm $(\mathrm{P}>0.05)$, as

shown in Fig 8.

Fig 8. Mean change in respiration rates $^{1}$ across three phases in non-conditioned Labradors.

No effect of coat color $(\mathrm{P}=0.5560)$ or sex $(\mathrm{P}=0.9806)$ was seen for water consumption

with black dogs consuming $173.75 \pm 195.3 \mathrm{ml}$ and yellow dogs consuming

$221.21 \pm 57.1 \mathrm{ml}$.

No effect of coat color was noted when rectal temperatures were examined for a return to

baseline in $12.5 \%$ and $28.6 \%$ of black and yellow dogs respectively, $(\mathrm{P}=0.5692)$. Similarly, GI

temperatures returned to baseline $50 \%$ of black and $42.9 \%$ of yellow dogs $(\mathrm{P}=1.00)$ and

abdominal surface temperature with $12.5 \%$ black and $28.6 \%$ yellow dogs $(\mathrm{P}=0.6080)$ returning 
to baseline values. Conversely, coat color did impact the dog's return to baseline when eye surface temperature was examined with $12.5 \%$ black and $71.4 \%$ yellow dogs achieving baseline

values after their Cooling phase, as shown in Table $3(\mathrm{P}=0.0406)$.

Table 3. Return to baseline ${ }^{1}$ rectal $^{2}$, gastrointestinal ${ }^{3}$ (GI), thermal eye ${ }^{4}$, and thermal abdominal ${ }^{5}$ temperatures by coat color and sex.

271

\begin{tabular}{|l|l|l|l|l|l|l|}
\hline VARIABLE & BLACK & YELLOW & P-VALUE & MALE & FEMALE & P-VALUE \\
\hline RECTAL & $12.5 \%$ & $28.6 \%$ & 0.5692 & $16.7 \%$ & $22.2 \%$ & 1.00 \\
\hline GI & $50 \%$ & $42.9 \%$ & 1.00 & $33.3 \%$ & $55.6 \%$ & 0.6084 \\
\hline EYE & $12.5 \%$ & $71.4 \%$ & $0.0406^{*}$ & $50 \%$ & $33.3 \%$ & 0.2286 \\
\hline ABDOMINAL & $12.5 \%$ & $28.6 \%$ & 0.6080 & $0 \%$ & $33.3 \%$ & 0.6080 \\
\hline
\end{tabular}

272

273

274

275

276

277

278

279

280

281

282

283

288

289

*Notes a significant difference between groups

${ }^{1}$ Return to Baseline occurred when cooling temperature returned within $0.5 \circ \mathrm{F}$ of the initial baseline temperature reading measured 30 min prior to sunlight exposure (sunlight

${ }^{2}$ Rectal temperature was recorded by inserting a thermometer in $2 \mathrm{~cm}$ rectally

${ }^{3} \mathrm{GI}$ (gastrointestinal) temperature was recorded with an ingestible thermistor CorTemp 30 minutes prior to baseline

${ }^{4}$ Eye temperature was captured using thermography FLIR T400 at the left eye

${ }^{5}$ Abdominal temperature was captured using thermography FLIR T400 at the left caudal abdomen

Sex did not influence cooling as rectal temperatures returned to baseline in $22.2 \%$ and $16.7 \%$ of female and male dogs respectively $(\mathrm{P}=1.00)$. Temperatures for the GI tract returned to baseline in $55.6 \%$ of female and $33.3 \%$ of male dogs $(\mathrm{P}=0.6084)$.

Similarly, no effect of sex was observed for cooling of surface temperatures measured at the caudal abdomen with $33.3 \%$ of female and $0 \%$ of male dogs returning to baseline values (P $=0.6080$ ). Eye surface temperature returned to baseline in $33.3 \%$ female and $50 \%$ of male dogs achieving baseline values after their Cooling phase $(\mathrm{P}=0.2286)$, as shown in Table 3 .

\section{Discussion}

Black dogs did not demonstrate a difference in temperature following exposure to direct sunlight when compared to yellow dogs for any of the parameters we examined, 
292 including rectal thermometer using a standard medical-grade predictive digital thermometer,

293 GI temperature using an ingestible thermistor, eye surface temperature and abdominal

294 surface temperature using forward-looking infrared thermography, respiration or water

295 consumption. Contradictory to currently held beliefs, all dogs experienced a similar rise

296 in rectal, GI, surface temperature, and respiration from the baseline to the sunlight

297 phase, with no difference shown between dark vs lighter coated dogs $(\mathrm{P}>0.05)$ as shown

298 in Figures 3-5 and Table 1. Furthermore, no effect of sex was measured as both males

299 and females demonstrated similar responses to sunlight exposure and cooling based on

300 rectal, GI, eye surface, abdominal temperatures, and respiration rates $(\mathrm{P}>0.05)$ shown

301 in Figures 6-8, and Table 2.

302 Contrary to the commonly held belief, our data demonstrated that black dogs did not

303 experience a greater heat gain than their yellow counterparts. Similarly, there was no

304 difference in the apparent thermoregulatory effect between dark and light dogs. This is

305 particularly noteworthy because of the relative short duration of the walk and the significant

306 temperature increase we observed in both dark and light-coated dogs. It is also interesting to

307 note that the 15 -minute cooling period was inadequate for $80 \%$ of the dogs to achieve

308 baseline thermal status based on rectal measurements which are considered standard for

309 recording accurate temperature in animal species [16] .

310 Conversely, almost $50 \%$ of each group was able to return to a baseline values via

311 GI values after 15 minutes of cooling. This could be attributed to water consumption

312 during the cooling phase affecting the CorTemp capsule reading. [17] However, it is

313 important to note that all dogs did experience a significant decrease in their rectal, GI,

314 eye and abdominal temperatures, and respiration rates. Future work should include 
315 studies with a longer cooling period to determine that time frame necessary for

316 non-conditioned dogs to achieve baseline thermal status following thermal stress.

317 The data presented here are inconsistent with the previous study examining racing

318 greyhounds with larger proportions of dark coated dogs having higher rectal

319 temperatures after racing [5]. Key differences between this study and the prior study on

320 Greyhounds include controlled coat color (black or yellow vs. multiple light or dark

321 colors), tighter grouping of age and sex, and a controlled time period and consistent

322 environment. However, there were fewer dogs in our study compared to the study on

323 Greyhounds (16 vs. 229). Power calculations indicate that it would take more than 500 dogs

324 to adequately test this question using an alpha of 0.05 and $80 \%$ power. That number of dogs

325 is beyond our capacity. Furthermore, the greyhounds utilized in the previous study were

326 considerably more fit than the non-conditioned dogs used in our study. Fitness level can

327 impact thermal response as previously demonstrated in working canines [18, 19] and

328 should be examined as a controlled factor in future work.

Infrared thermal cameras have been widely used in livestock species to identify

330 changes in the surface temperature of the animals. These studies focused on areas that

331 had more skin exposure for more accurate data, such as the flank, eye, and facial region.

332 In our canine study, thermal images were captured inside a building to reduce effects

333 from wind, sun, and other environmental exposures. Both yellow and black dogs showed

334 similar changes in body surface temperatures which does not support the idea that coat

335 color is a potential risk factor for thermal stress. A comparison of skin surface

336 temperature during exposure to sunlight in dogs is warranted. There were some

337 challenges associated with the capture of the thermal imaging. Although the baseline 
photos were captured with little difficulty, many of the dogs were hot following the sunlight exposure and several were non-compliant in assuming the same posture. More obedient/compliant dogs would prove better subjects for this nature of study. were similar between the dark and light coated dogs, perhaps dark coated dogs simply undertook increased efforts of thermoregulation such as increased respiration (i.e.

344 panting) or increased water consumption. However, we found no difference in these 345 parameters between the dark and light coated dogs, suggesting that the effort they expended to thermoregulation was also similar. A key limitation in our study is that we did not record heart rate, which would be important in assessment of thermoregulatory response. More sophisticated instrumentation and monitoring would be important in further studies to determine if more subtle physiological changes were occurring with thermoregulation.

Novel data produced by this work include an absence of significant difference in

352 body temperature between black or yellow coated dogs. The techniques utilized to assess

353 temperature, panting and water consumption are non-technical, readily available methods

354 for canine handlers or owners to assess thermal status of dogs in the field, and thus are 355 important to prevention of heat related injury. These data provide critical evidence to dispute 356 the theory that dark coat color is a risk factor for thermal stress which is reported across 357 several forums including veterinary textbooks and previously published articles [1-4].

358 In this experiment, dark and light-colored dogs exposed to the same environment 359 showed a similar heat gain and loss (mean peak rectal $40.31 \pm 0.37{ }^{\circ} \mathrm{C}$ and mean peak GI 
361 conditioned dogs reached rectal temperatures near $40.9{ }^{\circ} \mathrm{C}\left(105.62{ }^{\circ} \mathrm{F}\right)$, and

362 gastrointestinal temperatures near $42^{\circ} \mathrm{C}\left(106.16^{\circ} \mathrm{F}\right), 15$ minutes of rest in a cool room

363 with water available for consumption is adequate to begin to decrease the temperature,

364 although a return to baseline values may not be achieved within that time frame. No

365 medical intervention or active cooling method was needed to decrease the dogs'

366 temperature and nor heat-related negative health impacts were noted by the veterinary

367 team on site, despite dogs reaching temperatures as high as $42^{\circ} \mathrm{C}(106.16 \mathrm{~F})$.

369 Ethical Conflicts

370 The authors declare that they had no conflict of interest.

\section{Acknowledgements}

372 The authors would like to thank the undergraduate and graduate students of Southern Illinois

373 University for their participation in this research. The researchers would also like to

374 acknowledge SIT Service Dogs for contributing the use of their dogs for this project.

\section{Funding}

376 This study was partially supported by the USDA-ARS Biophotonics (Grant \# 58-6402-

377 3-018).

\section{References}

380 1. Flournoy S, Wohl J, Macintire D. Heatstroke in Dogs: Pathophysiology and Predisposing

$381 \quad$ Factors. Compend. 2003;25:410-418. 
382 2. Johnson SI, McMichael M, White G. Heatstroke in small animal medicine: A clinical

383 practice review. J Vet Emerg Crit Care. 2006;16(112-119):10-1111.

384 3. Andress M, Goodnight M. Heatstroke in a Military Working Dog. United States Ary Med

385 Dep Journal. 2013; p. 34-37.

386 4. Carter AJ, Hall EJ. Investigating factors affecting the body temperature of dogs

387 competing in cross country (canicross) races in the. UK J Therm Biol. 2018;72(33-

$388 \quad 38): 10-1016$.

389 5. McNicholl J, Howarth GS, Hazel SJ. Influence of the Environment on Body Temperature $390 \quad$ of Racing Greyhounds. Front Vet Sci. 2016;3(1-13):10-3389.

391 6. Chesney CJ. The microclimate of the canine coat: The effects of heating on coat and skin 392 temperature and relative humidity. Vet Dermatol. 1997;8:183-190.

393 7. Finch VA, Bennett IL, Holmes CR. Coat colour in cattle: effect on thermal balance, 394 behaviour and growth, and relationship with coat type. J Agric Sci. 1984;102(141).

395 8. Hillman PE, Lee CN, Carpenter JR, Baek KS, Parkhurst A. Impact of Hair Color on 396 Thermoregulation of Dairy Cows to Direct Sunlight; ASAE Annual Meeting. 2001.

397 9. Church JS et al. Influence of environmental factors on infrared eye temperature 398 measurements in cattle. Res Vet Sci. 2014;96(220-226):10-1016.

399 10. McManus C. et al., Infrared thermography in animal production: An overview. Comput $400 \quad$ Electron Agric. 2016;123:10-16.

401 11. Salles MSV, C S. Mapping the body surface temperature of cattle by infrared 402 thermography. J Therm Biol. 2016;62(63-69):10-1016.

403 12. Rizzo M, Arfuso F, Alberghina D, Giudice E, Gianesella M, Piccione G. Monitoring 404 changes in body surface temperature associated with treadmill exercise in dogs by use of 
infrared methodology. J Therm Biol. 2017;69(64-68):10-1016.

406 13. Zanghi BM. Eye and Ear Temperature Using Infrared Thermography Are Related to

Rectal Temperature in Dogs at Rest or With Exercise. Front Vet Sci. 2016;3(111).

408 14. Yanmaz LE, Doğan E, Okumuş Z, Şenocak MG, Yildirim F. Comparison of Rectal, Eye 617):10-9775.

411 15. Gomart SB, Allerton FJW, Gommeren K. Accuracy of different temperature reading techniques and associated stress response in hospitalized dogs; Journal of Veterinary Emergency and Critical Care. 2014;24:279-285

414 16. Greer RJ, Cohn LA, Dodam JR, Wagner-Mann CC, Mann FA. Comparison of three methods of temperature measurement in hypothermic, euthermic, and hyperthermic dogs. J Am Vet Med Assoc. 2007;230:1841-1848.

417 17. Lewis S, Foster RC. Effect of Heat on Canines and Felines. Iowa State Univ Veterinarian. 1976;38:117-121.

419 18. Robbins PJ, Ramos MT, Zanghi BM, Otto CM. Environmental and Physiological Factors Associated with Stamina in Dogs Exercising in High Ambient Temperatures. Front Vet Sci. 2017;4(1-9):10-3389.

422 19. Baker JA, Davis MS. Effect of conditioning on exercise-induced hyperthermia and postexercise cooling in dogs. Comp Exerc Physiol. 2018;14(91-97):10-3920. 
Fig 3. Mean change in rectal ${ }^{1}$ and gastrointestinal temperature $(\mathrm{GI})^{2}$ across three phases in non-conditioned Labradors.

\section{Impact of coat color across phase on rectal and gastrointestinal temperature}

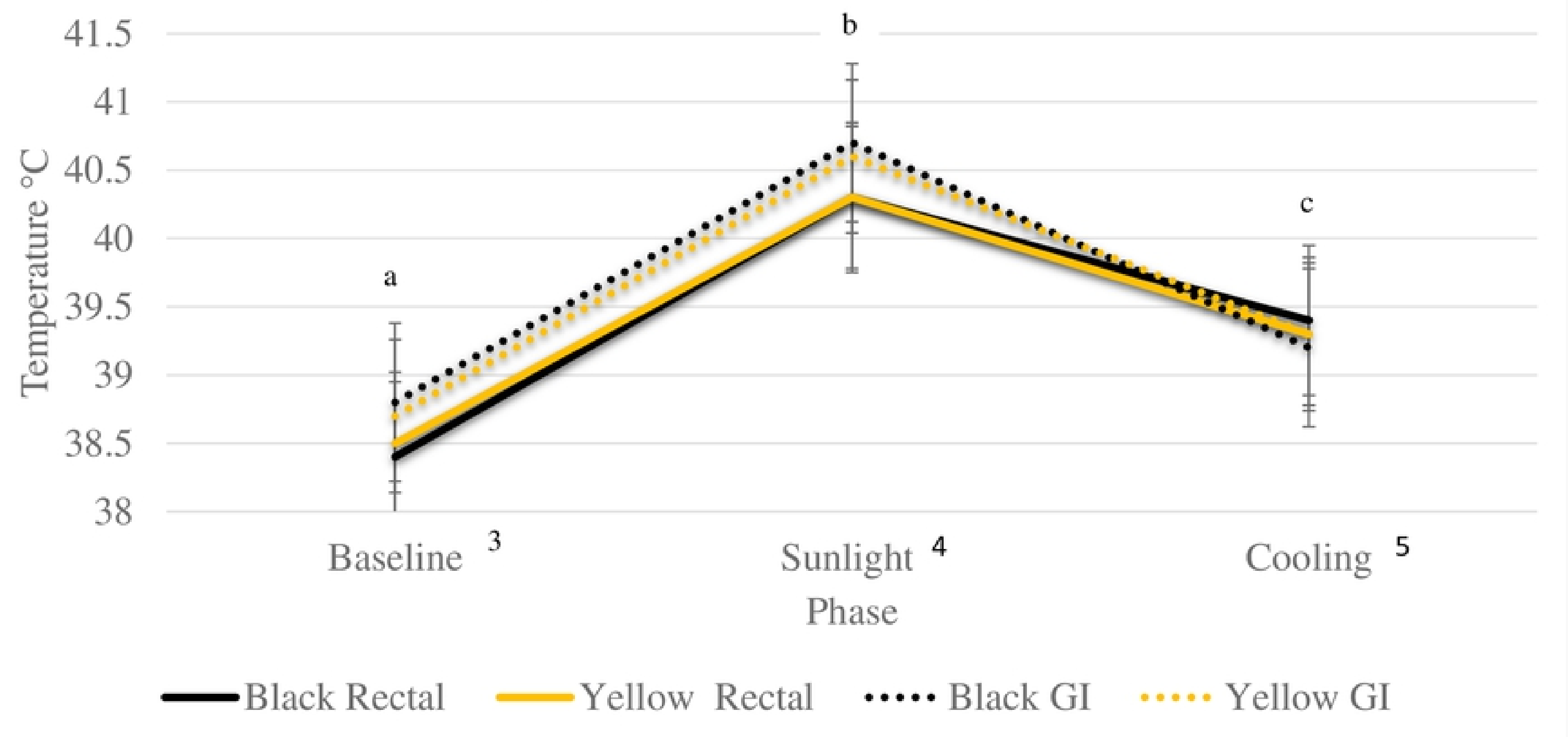

a,b,c Notes significant difference by phase

"Notes significant difference by color

${ }^{1}$ Rectal temperature was recorded by inserting thermometer to a depth of approximately $2 \mathrm{~cm}$ with petroleum jelly

${ }^{2} \mathrm{GI}$ (gastrointestinal) temperature was recorded with an ingestible thermistor CorTemp 30 minutes prior to baseline

${ }^{3}$ Baseline occurred 30 minutes prior to sunlight exposure (sunlight) and recorded initial measurements

${ }^{4}$ Sunlight phase consisted of 30 minutes of active walking in a sunny outdoor area on a leash

${ }^{5}$ Cooling phase occurred 15 minutes after walking in a climate-controlled room with water

Figure 3 
Fig 4. Mean change in body surface temperature measured by thermography ${ }^{1}$ at the eye ${ }^{2}$ and abdomen ${ }^{3}$ in non-conditioned Labradors

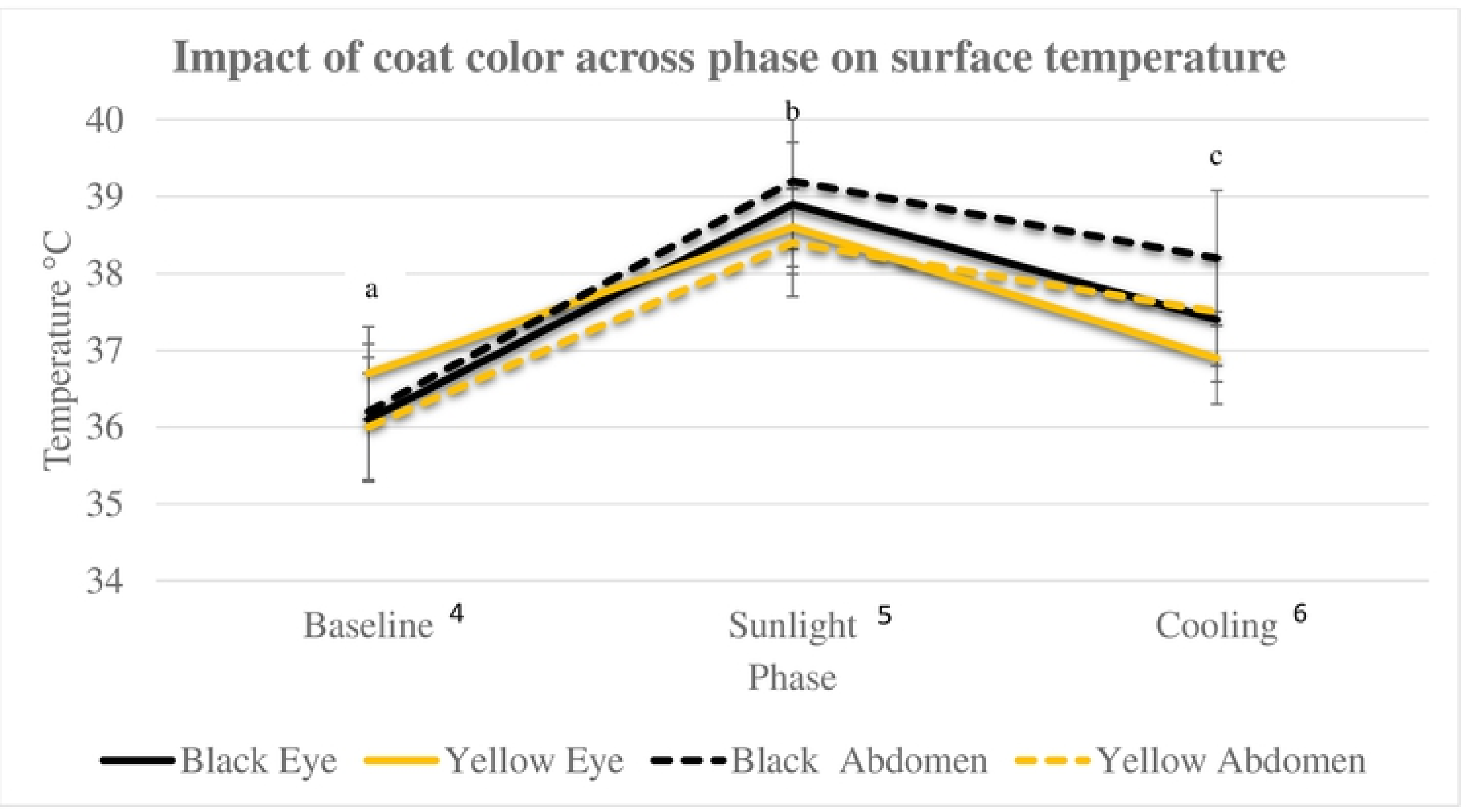

$\mathrm{a}, \mathrm{b}, \mathrm{c}$ Notes significant difference by phase

Notes significant difference by coat color

IThermography captured using FLIR T400

${ }^{2}$ Eye temperature was captured using thermography focusing on left eye

${ }^{3}$ Abdomen $(\mathrm{Ab})$ temperature was captured using thermography focusing on left caudal abdomen

${ }^{4}$ Baseline occurred 30 minutes prior to sunlight exposure (sunlight) and recorded initial measurements

${ }^{5}$ Sunlight phase consisted of 30 minutes of active walking in a sunny outdoor area on a leash

${ }^{6}$ Cooling phase occurred 15 minutes after walking in a climate-controlled room with water

Figure 4 
Fig 5. Mean change in respiration rates ${ }^{1}$ across three phases in non-conditioned Labradors.

\section{Impact of coat color across phase on respiration rate}
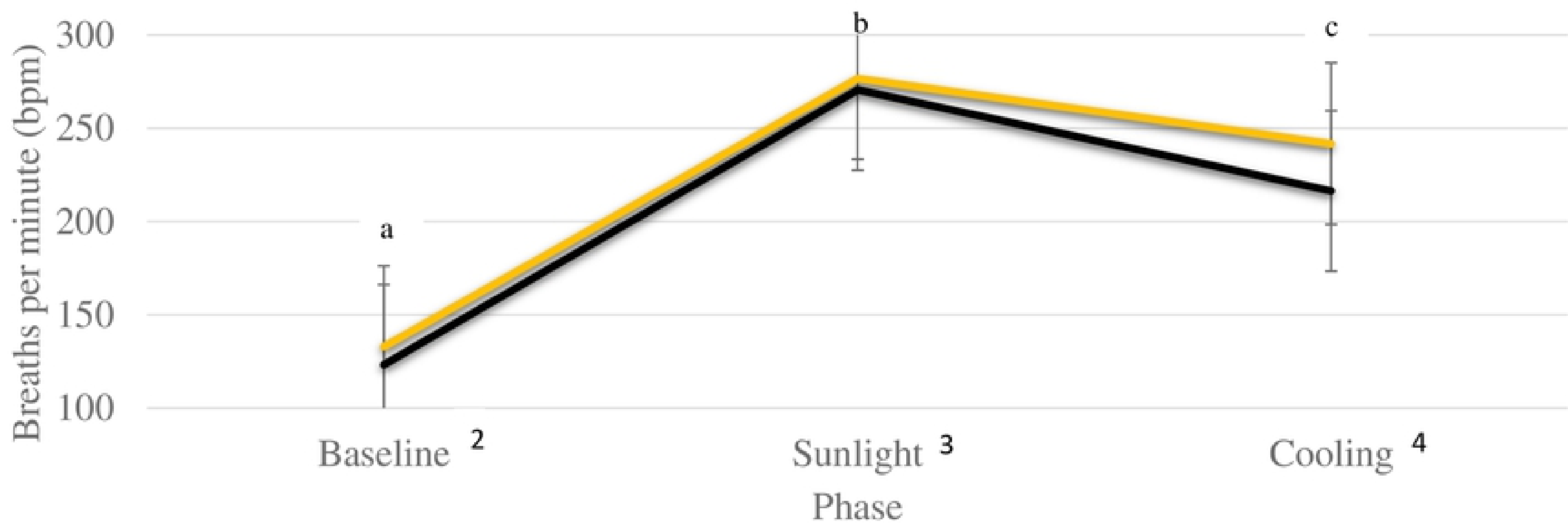

-Black Respiration

-Yellow Respiration

a,b,c Notes significant difference by phase

*Notes significant difference by coat color

${ }^{1}$ Respiration rate was captured for 30 seconds by a GoPro camera

${ }^{2}$ Baseline occurred 30 minutes prior to sunlight exposure (sunlight) and recorded initial measurements

${ }^{3}$ Sunlight phase consisted of 30 minutes of active walking in a sunny outdoor area on a leash

${ }^{4}$ Cooling phase occurred 15 minutes after walking in a climate-controlled room with water

Figure 5 
Fig 6. Mean change in rectal ${ }^{1}$ and gastrointestinal temperature $(\mathrm{GI})^{2}$ across three phases in non-conditioned Labradors.

\section{Impact of sex across phase on rectal and gastrointestinal temperature}

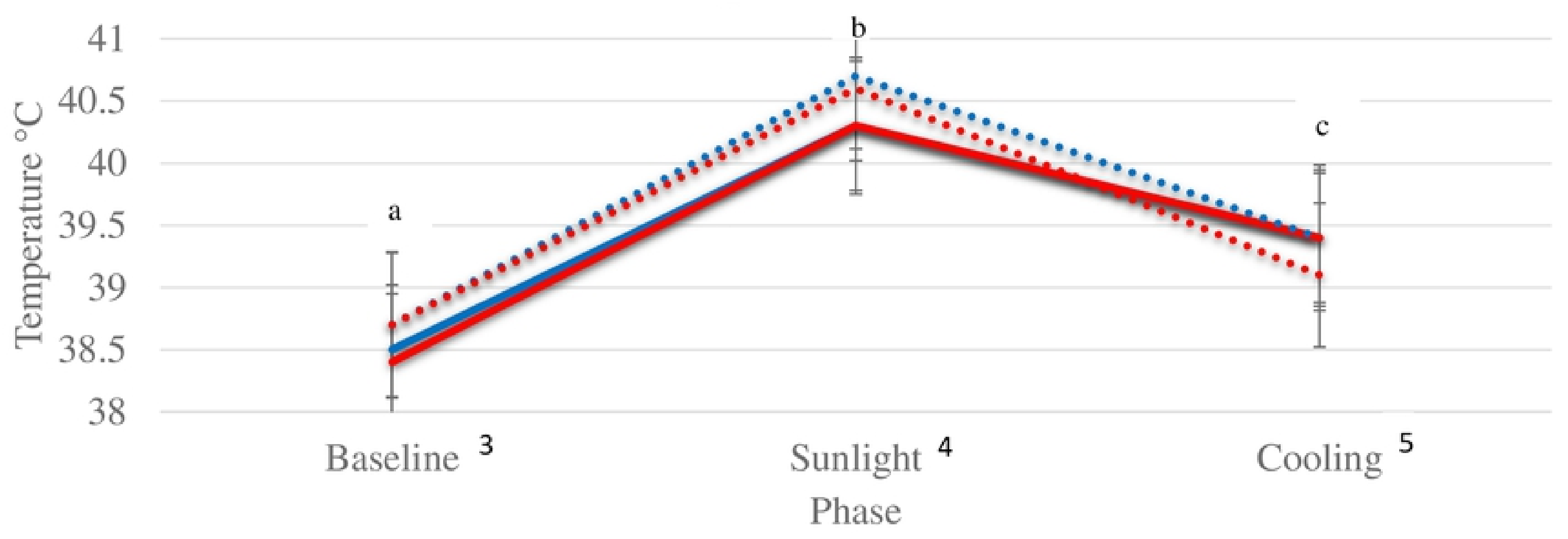

\section{—Male Rectal —Female Rectal $\quad \cdots .$. Male GI $\quad \cdots .$. Female GI}

a.b.c Notes significant difference observed by phase

Notes significant difference observed by sex

1 Rectal temperature was recorded by inserting thermometer to a depth of approximately $2 \mathrm{~cm}$ with petroleum jelly

$2 \mathrm{GI}$ (gastrointestinal) temperature was recorded with an ingestible thermistor CorTemp administered 30 minutes prior to baseline

${ }^{3}$ Baseline occurred 30 minutes prior to sunlight exposure and recorded initial measurements

4 Sunlight phase consisted of 30 minutes of active walking in a sunny outdoor area on a leash

${ }^{5}$ Cooling phase occurred 15 minutes after walking in a climate-controlled room with water

Figure 6 
Fig 7. Mean change in surface temperature measured by thermography ${ }^{1}$ at the eye ${ }^{2}$ and abdomen ${ }^{3}$ in non-conditioned Labradors across three phases.

\section{Impact of sex across phase on surface temperature}

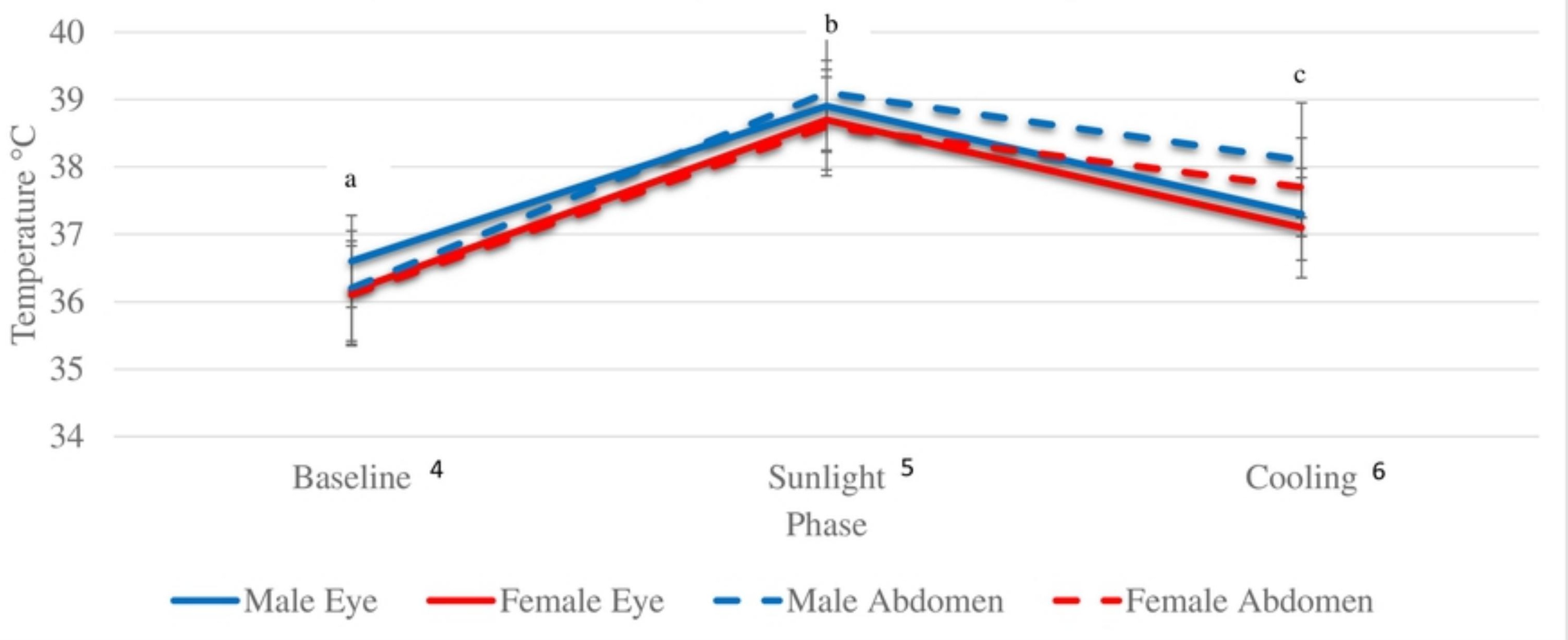

ab,c Notes significant difference observed by phase

Notes significant difference observed by sex

'Thermography captured using FLIR T400

${ }^{2}$ Eye temperature was captured using thermography focusing on left eye

${ }^{3} \mathrm{Abdomen}(\mathrm{Ab})$ temperature was captured using thermography focusing on left caudal abdomen

'Baseline occurred 30 minutes prior to sunlight exposure and recorded initial measurements

S Sunlight phase consisted of 30 minutes of active walking in a sunny outdoor area on a leash

${ }^{6}$ Cooling phase occurred 15 minutes after walking in a climate-controlled room with water

Figure 7 
Fig 8. Mean change in respiration rates ${ }^{1}$ across three phases in non-conditioned Labradors.

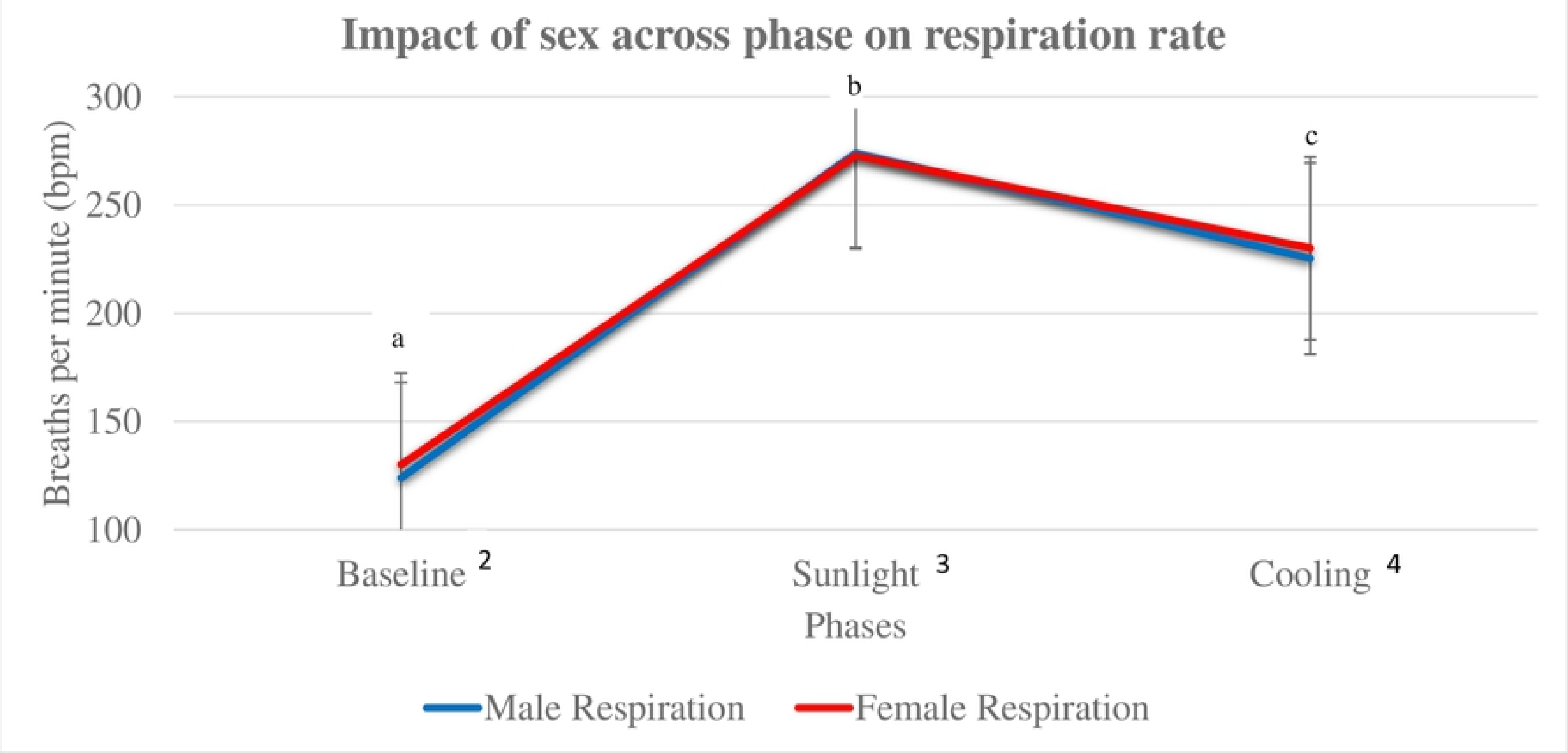

a.b,c Notes significant difference observed by phase

"Notes significant difference observed by sex

${ }^{1}$ Respiration rate was captured for 30 seconds by a GoPro camera

${ }^{2}$ Baseline occurred 30 minutes prior to sunlight exposure and recorded initial measurements

${ }^{3}$ Sunlight phase consisted of 30 minutes of controlled pace walking in a sunny outdoor area on a leash

${ }^{4}$ Cooling phase occurred 15 minutes after walking in a climate-controlled room with water

Figure 8 